\title{
Establishing jet scaling patterns with a photon
}

\author{
Christoph Englert, ${ }^{a}{\text { Tilman Plehn, }{ }^{a} \text { Peter Schichtel }}^{a}$ and Steffen Schumann ${ }^{a, b}$ \\ ${ }^{a}$ Institut für Theoretische Physik, Universität Heidelberg, \\ Philosophenweg 16, 69120 Heidelberg, Germany \\ ${ }^{b}$ II. Physikalisches Institut, Universität Göttingen, \\ Friedrich-Hund-Platz 1, 37077 Göttingen, Germany \\ E-mail: c.englert@thphys.uni-heidelberg.de, plehn@uni-heidelberg.de, \\ p.schichtel@thphys. uni-heidelberg.de, \\ steffen.schumann@phys. uni-goettingen.de
}

ABSTRACT: Staircase and Poisson scaling are two typical patterns we observe for the exclusive number of jets at high energy hadron colliders. We examine these scaling properties for photon plus jets production at the LHC and find that this channel is well suited to study these features. We illustrate and discuss when to expect each of the two patterns, how to induce a transition through kinematic cuts, and how photons are related to heavy gauge bosons. Measurements of photon+jets production is therefore providing valuable information on exclusive jet scaling, which is going to help to eventually understand the theoretical origin of exclusive jet scaling properties in more detail.

Keywords: Jets, Hadronic Colliders, Standard Model 


\section{Contents}

1 Introduction 1

2 Staircase scaling $\quad 3$

$\begin{array}{lll}3 & \text { Poisson scaling } & 6\end{array}$

4 Massive gauge bosons $\quad 8$

5 Outlook $\quad 9$

\section{Introduction}

After testing and reproducing many interesting aspects of the Standard Model at the LHC, the focus of the ATLAS and CMS collaborations is rapidly moving toward searches for Higgs particles [1, 2] or physics beyond the Standard Model [3]. The production rates for any of these search channels are small, for example compared to $W / Z+$ jets or top pair production, channels which constitute their main backgrounds. Traditionally, at hadron colliders we have relied on the appearance of leptons, photons or missing transverse energy to point us to interesting new physics processes. In this approach QCD effects and jet production are either ignored or considered a nuisance.

Starting with the suggested searches for a light Higgs boson in weak boson fusion [4-6], this attitude has changed; this search shows how the QCD structure of signal events can be turned into a powerful handle to reject large backgrounds. The key analysis tools are (central) jet vetos [7-13], which for example suppress QCD-initiated $W / Z+$ jets events or hadronic top pair production. Implicitly, this approach is adopted in Higgs searches for example in the $H \rightarrow W W$ or $H \rightarrow \gamma \gamma$ channels, when those searches are divided into 0-jet, 1 -jet and soon 2-jet strategies $[1,2]$.

In a similar spirit, searches for example for supersymmetry benefit from the measurement of the number of jets which includes information on the color structure of the new heavy states [14]; the only caveat is that we need to carefully separate decay jets from QCD jet radiation associated with hard processes [14-17]. What is missing for all such analyses is an experimentally established and theoretically sound link between choosing $n$-jet samples for an analysis and a systematic study of the corresponding $n_{\text {jets }}$ distribution for signal and background processes [12]. A dedicated study along this line would map out the behavior of exclusive $n_{\text {jets }}$ distributions after different cuts, understand its basic features, and quantify the notorious theory uncertainties associated with jet counting. As it will turn out, the production of a photon in association with QCD jets is a perfect basis for such studies and complements the ones, which have already been performed at the LHC [18]. 
From text book quantum field theory we know that successive $p_{T}$-ordered photon radiation off a hard electron - as well as successive gluon radiation off a hard quark - follows a Poisson pattern for the exclusive number of photons or gluons [19]. This pattern corresponds to a simple probabilistic picture of successive independent splitting. The splitting probability is linked to the coupling constant, the color factor, the form of the splitting kernel, and a scale logarithm. The Poisson scaling pattern, however, has not been observed in inclusive production at hadron colliders since UA1/UA2. Instead, for many processes we find staircase scaling, namely a constant ratio of exclusive $n$-jet rates $\sigma_{n+1} / \sigma_{n}=R$ [20-22]. This feature has been studied by the LHC experiments [23] and also finds application in background-modelling in phenomenological approaches [24, 25].

The description of exclusive jet rates is at odds with our description of QCD at hadron colliders. Parton densities obeying the DGLAP equation [26-28] resum collinear logarithms and absorb the corresponding infrared divergences. As a consequence, any computation based on such parton densities is jet inclusive, i.e. it allows for an unspecified number of collinear jets radiated off the incoming partons. Strictly following the DGLAP approximation, these perfectly collinear jets are not observable at the LHC. However, the assumption of perfect collinearity is modified by the initial state parton shower, which redistributes the dominantly collinear jet radiation into the physical phase space. Evaluating exclusive event samples with exactly $n$ jets is theoretically limited by the precision of the parton shower description, including its obvious breakdown for sizeable transverse momenta [29]. This is the reason why in the past exclusive $n_{\text {jets }}$ distributions could rarely be exploited to compare collider data to QCD predictions. However, as described above, current LHC analyses force us to overcome this limitation and study exclusive $n_{\text {jets }}$ distributions (including cuts corresponding to jet veto survival probabilities) starting from perturbative QCD.

Matching of a hard QCD matrix element with a collinear parton shower [30-37] allows us to reliably simulate and study jet scaling patterns from perturbative QCD. It does not require a fundamental re-organization of QCD perturbation theory [38-40] but simply relies on the proper phase space simulation of collinear logarithms. This way it does not only include the radiation of one or two hard jets, as correctly described by fixed-order QCD calculations, but any number of radiated jets including high multiplicities obviously well described by the parton shower. We use Sherpa [41-43] with its CKkw [34, 35] matching scheme to describe radiation of up to seven jets with high precision. Typically, we check an additional two more jets for unexpected features, but with correspondingly reduced statistics.

As we will show in this paper, a particularly promising channel to measure exclusive jet rates and compare them to QCD predictions is hard photon production in association with jets.

The cross section is large enough to already have enough data to not only validate Monte Carlos, but also test potential $n_{\text {jets }}$ scaling hypotheses in various phase space regions. We will show how to define and extract different kinematical regimes of the photon to compare various hypotheses of QCD radiation with data and to cross check jet emission against other production modes. Possible applications towards new physics searches under the premise that Poisson or staircase scaling are phenomenologically realized to good approximation have been discussed in refs. [1, 2, 7-12]. 


\section{Staircase scaling}

Some qualitative implications of staircase scaling are already known from the analysis of the inclusive $n_{\text {jets }}$ distribution of Standard Model processes. These include pure QCD jets production $[14,44-49]$ and $W / Z$ production with jets [20-22, 44-49]. As we will see, $\gamma+$ jets production when constrained in certain cut scenarios provides an additional channel, hence contributing to a better understanding of the possibly observed $n_{\text {jets }}$ scaling patterns. Staircase scaling is defined in terms of constant ratios of the experimentally measured jet-inclusive $n$-jet cross sections

$$
\hat{R}_{(n+1) / n}=\frac{\hat{\sigma}_{n+1}}{\hat{\sigma}_{n}} \equiv \hat{R} . \quad \text { (jet-inclusive) }
$$

It turns out that we can equivalently formulate this condition in terms of inclusive $(\hat{\sigma})$ and exclusive $(\sigma)$ numbers of jets. Correspondingly defining

$$
R_{(n+1) / n}=\frac{\sigma_{n+1}}{\sigma_{n}} \equiv R \quad \text { (jet-exclusive) }
$$

for the exclusive rates, the resulting ratios are identical [14],

$$
\hat{R}=\frac{\sum_{j=n+1} \sigma_{j}}{\sigma_{n}+\sum_{j=n+1} \sigma_{j}}=\frac{\sigma_{n+1} \sum_{j=0}^{\infty} R^{j}}{\sigma_{n}+\sigma_{n+1} \sum_{j=0}^{\infty} R^{j}}=\frac{R \sigma_{n} \frac{1}{1-R}}{\sigma_{n}+R \sigma_{n} \frac{1}{1-R}}=R .
$$

This way the merits of perturbative QCD and its perturbative predictions directly translate to the jet-exclusive final states in a well-defined approach where lower multiplicities are utilized to constrain the higher ones.

While a proper analytical derivation of this feature from first principles is still missing, it is observed to a good approximation in both experimental data and theoretical calculations using matrix element and parton shower merging [44-51]. Only using jet radiation via parton showering this scaling feature is not matched as well, which is expected given that such inclusive processes do not offer a hard scale in relation to which we can define collinear radiation. In addition, for $W+$ jets production it has been shown that fixed order QCD corrections stabilize the observed staircase pattern [52].

Unlike the other cases mentioned above, at first sight photon production does not posses an obvious jet scaling behavior. It only occurs once we include strong separation cuts between the photon and each of the jets, effectively removing any logarithmic enhancement linked to QED photon radiation. Moreover, as we will show in this paper, different basic cuts can easily induce different scaling patterns.

For our simulation we rely on SHERPA v1.3.0 [41-43] and its CKKW matching up to five matrix element jets. We reconstruct jets using the anti- $k_{T}$ algorithm from FASTJET [53$55]$ with $R_{\text {anti-k }}=0.4$, which gives us a very moderate geometric separation of two jets. When dealing with photons in a QCD environment some familiar subtleties have to be considered [56-61]: a photon can arise from non-perturbative fragmentation. Those photons are not useful in our case since our focus is obviously not on QED corrections to 

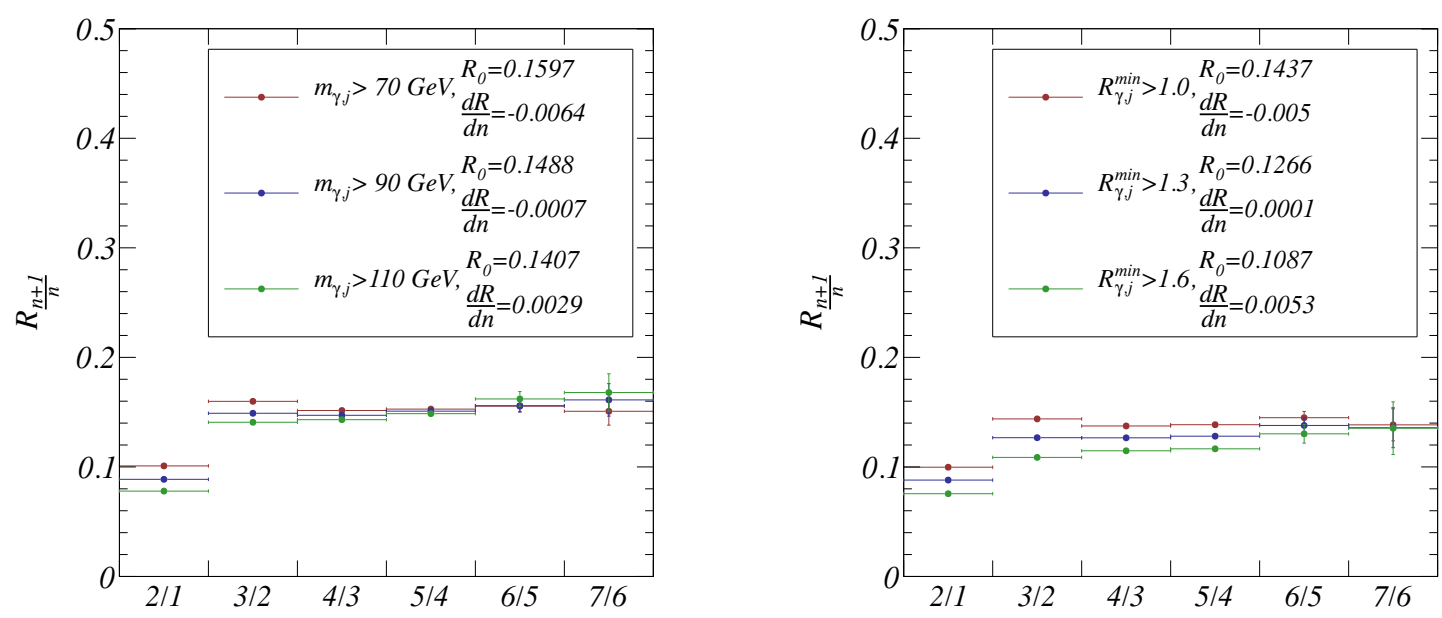

Figure 1. Two scenarios establishing staircase scaling for $\gamma+$ jets production at the $7 \mathrm{TeV}$ LHC. Left: invariant mass criterion of $m_{\gamma j}>70,90,110 \mathrm{GeV}$ for each jet. Right: geometric separation between the photon and each of the jets. The extracted values for $R_{0}$ and $d R / d n$ are defined in eq.(2.5). The error bars correspond to our numerics with $1.6 \cdot 10^{7}$ events.

multi-jet production rates. Therefore, we opt for a solid photon isolation. A naive hard cut e.g. on the jet-photon $R$ distance limits the phase space of soft gluon emission and is infrared unsafe. We instead define an isolated photon through a hadronic energy deposit of less than $10 \%$ of $p_{T, \gamma}$ in a cone of size $R<0.4$ centered around the photon direction [56-58]. If this criterion is not met the photon candidate is pushed into the jet finding algorithm.

For reconstructed jets and photons in this section we then require

$$
p_{T, \gamma}>50 \mathrm{GeV}, \quad p_{T, j}>50 \mathrm{GeV}, \quad\left|\eta_{\gamma}\right|<2.5, \quad\left|y_{j}\right|<4.5,
$$

where $\eta$ and $y$ denotes the pseudo-rapidity and rapidity, respectively. These cuts are very inclusive and democratic, so we can expect to observe the staircase scaling behavior known for pure QCD jets.

We observe that photon plus jets events fulfilling eq.(2.4) alone do not show any kind of simple jet scaling behavior. What we are still missing is the crucial photon-jet separation criterion. In figure 1 we show two sets of $n_{\text {jets }}$ distributions for different separation criteria. Inspired by the $W / Z+$ jets analysis we can define a wide photon-jet separation in terms of the invariant mass. In figure 1 we find that almost prefect staircase scaling appears for minimal values of $m_{\gamma j} \gtrsim m_{Z}$, with a very slight degradation for alternative mass scales. The corresponding description in terms of a geometric separation leads to very similar results, but only once we require $R_{\gamma, j}>1$. This value we can understand from the typical $m_{\gamma j}$ values combined with $p_{T}>50 \mathrm{GeV}$. In both cases the first ratio $R_{2 / 1}$ is notorious, an effect that has been observed in other channels before [14]. The origin lies in a strong PDF suppression of the two-jet configurations. The price to pay for producing an additional jet, passing in particular the $p_{T, j}$ criterion, is highest for the transition $\sigma_{\gamma j} \rightarrow \sigma_{\gamma j j}$. The measure is the relative increase in partonic center-of-mass energy in order to produce the 

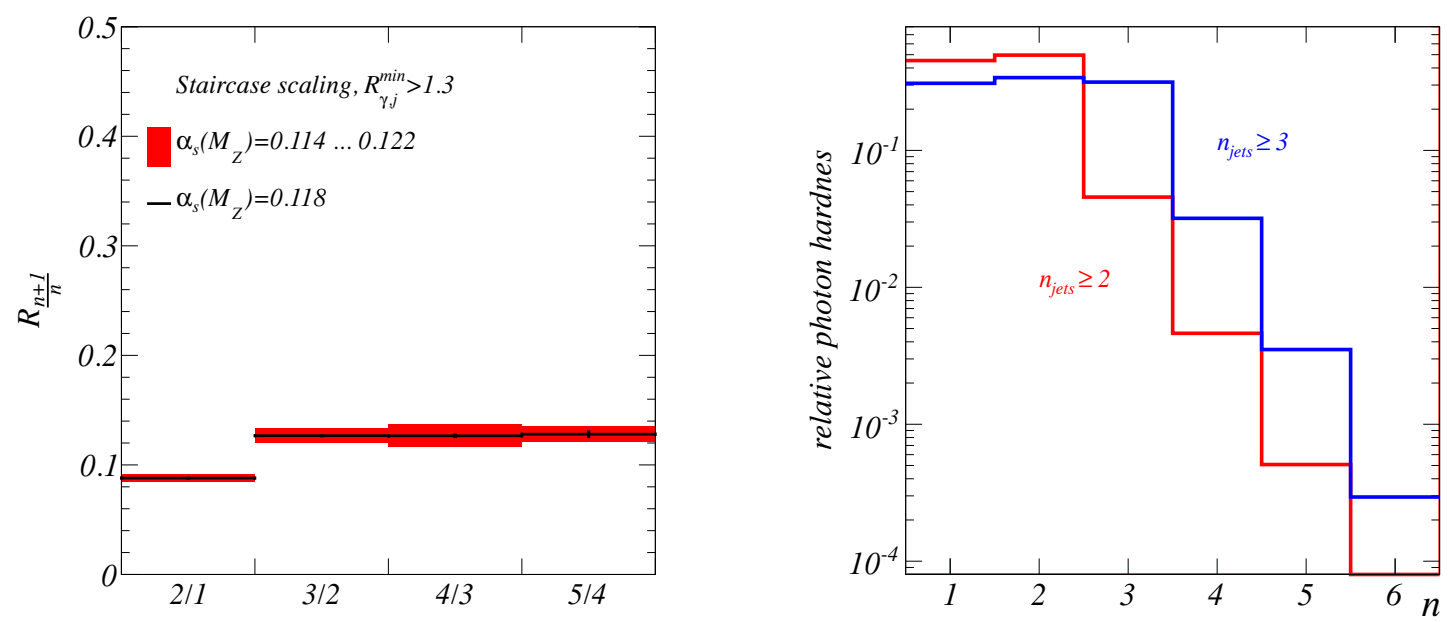

Figure 2. Left: effect of a consistent variation of $\alpha_{s}\left(M_{z}\right)$ on staircase scaling. Right: position of the photon in comparison to all jets, ordered by $p_{T}$. The two curves are for at least two-jet and at least three-jet events.

final state with one additional jet. This relative increase and the corresponding PDF suppression factor, however, becomes rather insignificant for higher jet multiplicities.

Setting aside the first entry we can test the quality of staircase scaling by fitting the form

$$
R_{(n+1) / n}=\frac{\sigma_{n+1}}{\sigma_{n}}=R_{0}+\frac{d R}{d n} n,
$$

and determine the slope to compare it to the perfect staircase scaling prediction $d R / d n=0$. For all curves shown in figure 1 we find $d R / d n$ in the $0.01-0.001$ range, essentially compatible with zero. The constant values $R_{0}$ range around 0.14 , but with a small spread. In section 4 we will contrast these values with the $W / Z+$ jets cases $[14,20-22]$. While the definition of the hard process does play a role in determining $R_{0}$, in section 3 we will see that the far dominant factor is $p_{T, j}^{\min }$ fixed in eq.(2.4).

In the left panel of figure 2 we show the effect of varying $\alpha_{s}$ on the observed staircase scaling pattern. Between the largest and lowest values of $\alpha_{s}$ there is a $7 \%$ difference. The effect of this shift on $R$ is correspondingly small. The reason is that $\alpha_{s}$ and for example the gluon parton densities are not independently extracted [62]. An increase in the value for the strong coupling is compensated by a decrease in the corresponding gluon density, postponing the expected blow up of the theory uncertainty to larger $n$ values than we can show in figure 2 .

On theoretical grounds, we can link staircase scaling to the presence of the large gluon self coupling, i.e. the non-abelian structure of QCD. In the absence of any hard scale from our process, relatively hard jets are still mostly generated through initial state radiation (ISR). Our simulation confirms that the final state radiation (FSR) cascades initiated by the core process jet and additional ISR jets generates the large and democratic jet multiplicities defining staircase scaling. This is illustrated in the right panel of figure 2 . The production of one hard jet with one hard photon is dominated by the partonic subprocess $q g \rightarrow q \gamma$. Unless 
we induce a hard scale by cuts, the evolution of the incoming quark or gluon is dominated by few and non-abelian splittings after the first hard ISR emission. Geometrically, for 2-jet events the quark-photon system typically recoils against the harder of the QCD jets. Only from the 3-jet configuration on we can simply split the ISR gluon, with a jet separation given by $R>0.4$ according to the jet algorithm. Correspondingly, in the right panel of figure 2 we see that the photon is typically as hard as the hardest jets: if we require at least two jets the photon is the hardest or second hardest object in roughly half of the events each and the third hardest object only in $\mathcal{O}(5 \%)$ of all events. For at least three jets in the final state the photon is equally likely to be the first, second and third hardest object. In other words, while one of the jets might usually recoil against a relatively hard photon, the additional jets responsible for the staircase scaling pattern are relatively soft. This is a result of splitting hard ISR gluons.

\section{Poisson scaling}

According to field theory text book knowledge radiating massless gauge bosons off, e.g., a hard fermion does not follow a staircase pattern [19]. Instead, successive soft radiation ordered in $p_{T}$ yields a Poisson distribution which can potentially be observed in the exclusive number of jets

$$
\sigma_{n}=\sigma_{0} \frac{e^{-\bar{n}} \bar{n}^{n}}{n !}
$$

where $\bar{n}$ is the expected number of emissions. As an example for such a process consider the exponentiable purely abelian contributions to multi-jet rates in $e^{+} e^{-}$collisions, see e.g. $[63,64]$. In this section, we construct a cut scenario, for which the approximation of subsequent soft emission is sufficiently realized to observe Poisson scaling in $\gamma+$ jets.

For the exclusive scaling ratios eq. (3.1) translates into

$$
R_{(n+1) / n}=\frac{\sigma_{n+1}}{\sigma_{n}}=\frac{\bar{n}}{n+1} .
$$

The assumptions entering the derivation of Poisson scaling are twofold: first, there should be one splitting function, for example the radiation of a photon or a gluon off a fermion. In the soft limit successive gauge boson radiation is automatically ordered by the emission angle. This way, we avoid combinatorial factors of the kind $n$ ! from differently ordered emission in the numerator. The crucial factor $1 / n$ ! in eq.(3.1) appears through the overcounting of the bosonic phase space. Poisson scaling is what one expects from a statistical point of view when we assign probabilities to statistically independent splittings. An example for this statistical treatment are Sudakov factors or collinear splitting probabilities following the DGLAP equation. The reason why solutions of the DGLAP equation show a Poisson behavior is that in its derivation we only take into account successive splittings of incoming partons on their way from the proton to the hard process. This is exactly what corresponds to a resummation of collinear logarithms and the removal of infrared divergences through the definition of scale dependent parton densities. The presence of a large kinematical logarithm makes parton shower simulations the appropriate tool to reproduce Poisson scaling. To force multi-jet scaling for example in association with a 

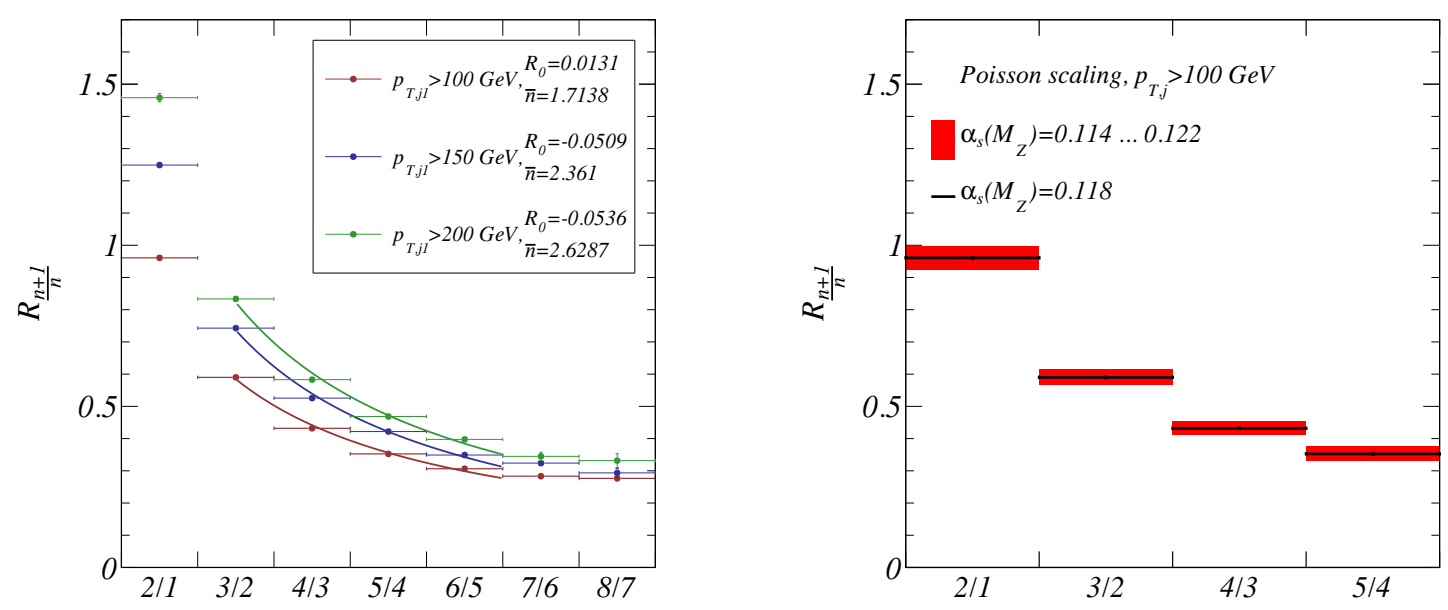

Figure 3. Poisson scaling for $\gamma+$ jets production at the $7 \mathrm{TeV}$ LHC. Left: different transverse momentum criteria for the leading jet, all other jets have $p_{T, j}>20 \mathrm{GeV}$. The extracted values for $R_{0}$ and $\bar{n}$ are defined in eq.(3.4). The error bars correspond to our numerics with $2.1 \cdot 10^{6}$ events. Right: effect of a consistent variation of $\alpha_{s}\left(m_{Z}\right)$.

photon into such a Poisson regime we can follow this argument of the parton shower and the Sudakov factors. What we need is a well defined hard subprocess, e.g. the leading 2-particle $\gamma$ - $j_{1}$ system which induces many successive splittings of the incoming partons.

Following the distance measures shown in figure 1 an obvious choice could be an increased value of $m_{\gamma j_{1}} \gg 100 \mathrm{GeV}$. This is similar to weak boson fusion Higgs production, where the large invariant mass of the two tagging jets $m_{j j}>600 \mathrm{GeV}$ induces a Poisson scaling of the $Z+$ jets backgrounds [12]. However, we find that the cleanest Poisson distribution is induced by requiring a single hard jet, i.e. requiring

$$
p_{T, \gamma}>20 \mathrm{GeV}, \quad p_{T, j}>100,20,20, \ldots \mathrm{GeV}, \quad\left|\eta_{\gamma}\right|<2.5, \quad\left|y_{j}\right|<4.5,
$$

instead of eq.(2.4). Generating the hard scale through the hardest jet is more efficient than asking for a hard photon, because according to our earlier argument a hard photon with $p_{T, \gamma}=100-200 \mathrm{GeV}$ could easily recoil against several jets from splitting ISR. Requiring a hard average $p_{T}$ for the jets would work as well, though. The important requirement is that through the cuts we induce a large scale separation with respect to the $p_{T, \text { min }}$ for radiated additional jets.

In figure 3 we see how enforcing a staggered $p_{T}$ cut scenario immediately changes the staircase scaling pattern into a Poisson distribution. Already for $p_{T, j_{1}}>100 \mathrm{GeV}$ we see a clear deviation from any kind of staircase behavior provided we allow all other jets to be as soft as $p_{T, j}>20 \mathrm{GeV}$. For $p_{T, j_{1}}>150 \mathrm{GeV}$ the leading $R_{2 / 1}$ ratio increases to values above unity, which means that in the exclusive $n_{\text {jets }}$ distribution the maximum will move away from zero.

To test the quality of the Poisson description we fit the $R_{(n+1) / n}$ distribution which is expected to follow eq.(3.2). If we allow for a deviation from the one-parameter Poisson 
shape of the kind

$$
R_{(n+1) / n}=\frac{\bar{n}}{n+1}+R_{0},
$$

$R_{0}$ is reminiscent of the staircase pattern eq.(2.3) and should come out essentially zero while $\bar{n}$ is the only free parameter in the Poisson shape and gives the expected number of jets. As expected, the value of $\bar{n}$ increases for harder leading jets. Just as for the staircase scaling we do not include the first entry $R_{2 / 1}$ in the fit.

While it follows the basic expectation, namely becoming large and exceeding unity, $R_{2 / 1}$ does not fit the Poisson shape well. In the first bin we encounter again a PDF suppression effect for producing the second jet. While this effect is much smaller than the mismatch of $R_{2 / 1}$ for staircase scaling, it is important to not include this bin into the fit to eq.(3.4) because it would lead to obviously wrong best-fit values for $\bar{n}$ and $R_{0}$.

If we also exclude the high-multiplicity bins we find small values of $\left|R_{0}\right|$, closer to zero than to $R_{7 / 6} \sim R_{8 / 7}$. This is expected. Our argument for Poisson scaling rests on the impact of a large scaling logarithm which has to be generated by successive and ordered ISR. Beyond some point these successive splittings will stop feeling a large logarithm and we can expect to fall back onto a non-negligible staircase scaling.

For example in the case of Higgs production we know that the large- $n$ limits of $R_{(n+1) / n}$ in the staircase and Poisson setups show hardly any difference. In figure 3 we estimate the staircase tail to be in the $R_{0} \sim 0.3$ range. This is significantly different from the $R_{0} \sim 0.15$ which we find in figure 1 . The reason is simply the reduced $p_{T}$ threshold of $20 \mathrm{GeV}$ for the Poisson studies. In the right panel of figure 3 we again show the consistent variation of $\alpha_{s}$. Clearly, the dependence is very small and does not affect the Poisson scaling feature.

\section{Massive gauge bosons}

Originally, jet scaling studies have been established for $W+$ jets events. Not surprisingly, $Z+$ jets events behave qualitatively and quantitatively the same [14]. The purpose of the first two sections of this paper is to show that after strict jet-photon isolation we can see the same scaling patterns in photon production.

Provided that for well separated photons the non-existence of large logarithms between inherent mass scales leads to staircase scaling we expect $\gamma+$ jets and $Z+$ jets production to be very similar. This very fact is also exploited experimentally to model the invisibly decaying $Z$ background to new physics searches [65] from corresponding measurements of $\gamma+$ jets in control regions [66-68]. In figure 4 we first see that based on the same jet cuts as in eq.(2.4) $Z$ production follows the same staircase pattern. The extracted value $R_{0}=0.149$ for $p_{T, j}>50 \mathrm{GeV}$ is consistent with the literature [14] as well with our findings in section 2 .

For the Poisson regime the situation is slightly different. For the photons a cut on the leading jet of $p_{T, j_{1}}>100 \mathrm{GeV}$ compared to a reduced general jet threshold of $20 \mathrm{GeV}$ already induces a large enough logarithm. The $Z$ mass in the final state could be expected to further enhance this scaling logarithm, even though it does not really translate into a collinear logarithm expressed in terms of $p_{T, j}$. In the right panel of figure 4 we find that the 

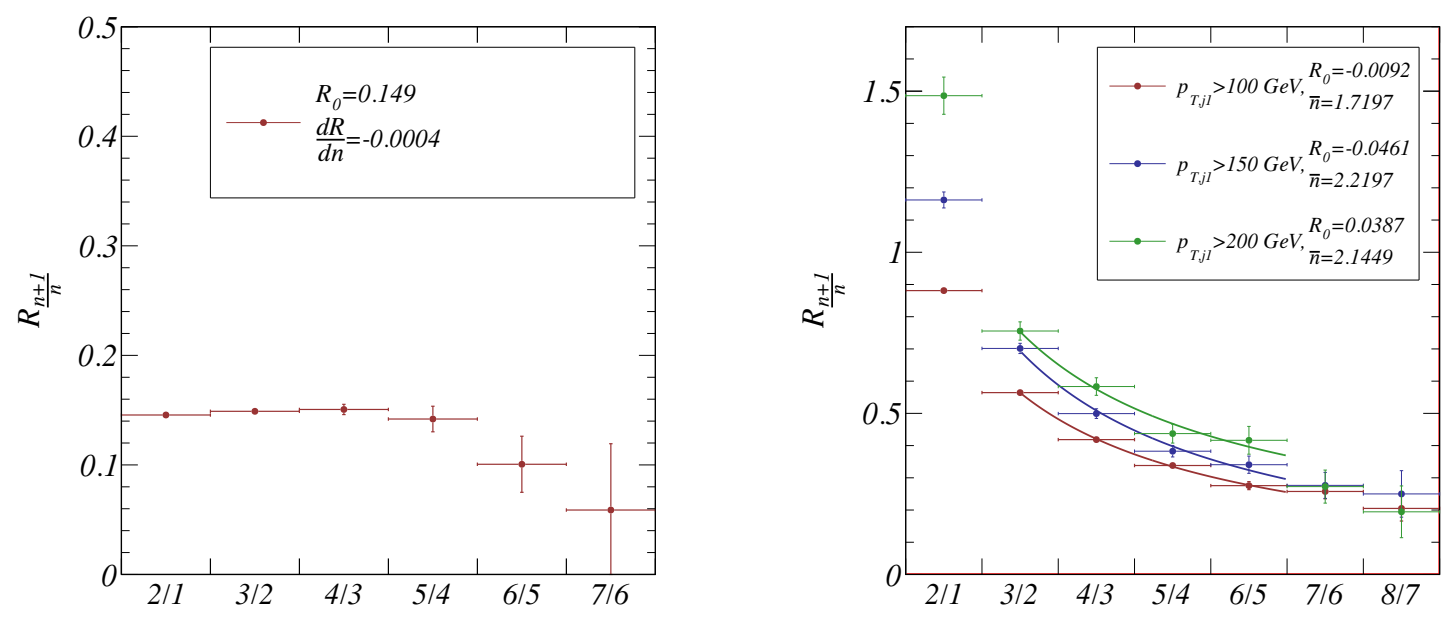

Figure 4. Scaling patterns for $Z+$ jets production at the $7 \mathrm{TeV}$ LHC. Left: staircase scaling for $p_{T, j}>50 \mathrm{GeV}$. Right: Poisson scaling for different transverse momenta of the hardest jet, all other jets have $p_{T, j}>20 \mathrm{GeV}$. The error bars correspond to our numerics with $10^{7}$ events before cuts.

$\bar{n}$ values we extract from the $Z$ case are very close to those for the photons, in particular taking into account the statistical uncertainties which affect the result for $p_{T, j_{1}}>200 \mathrm{GeV}$. The high-multiplicity staircase limit of the Poisson distribution for $R_{0} \sim 0.20-0.25$ again is similar to the photon case.

The agreement of both figures provides a consistency check of our previous statements but also opens up the possibility to experimentally cross check $Z+$ jets (and $W+$ jets) production against $\gamma+$ jets in yet another way. This holds not only on the qualitative but also on the quantitative level. The only difference between the two channels is that for photons the scaling patterns only appear once the photon is very well separated, controlling any additional QED logarithms which for the massive $Z$ case do not play any role at these energies.

\section{Outlook}

Counting numbers of exclusive jets has many applications in LHC searches, implemented for example as distinct Higgs analyses for different $n_{\text {jets }}$ values $[1,2]$ or central jet vetos [46]. To apply such cuts while maintaining stability of the theoretical predictions we need to properly understand exclusive $n_{\text {jets }}$ distributions both experimentally and theoretically. A major obstacle to overcome is that exclusive jet measurements have no straightforward interpretation in fixed order perturbation theory and exclusive quantities are typically plagued with large theoretical uncertainties. On the other hand, multi-jet merging, for example using the CKKW scheme $[34,35]$, allows us to study $n_{\text {jets }}$ distributions including a free choice of kinematic cuts and has proven successful in various experimental analyses so far.

Unlike $W / Z+$ jets and pure QCD jets production, the associated production of jets with a hard photon naively does not show simple scaling patterns. We show that once we 
require a widely separated photon we recover staircase scaling $\sigma_{n+1} / \sigma_{n}=R_{0}$ for the total cross section [14, 20-22].

Once we induce a large logarithm through kinematic cuts we see how the scaling pattern turns into a Poisson distribution for the exclusive number of jets. This is known for weakboson-fusion cuts in Higgs production [12]. For our photon channel a transverse momentum cut on the leading jet (and not on the photon) works best. Only in the high-multiplicity regime an underlying staircase pattern remains.

Given our observations and the large available photon sample at LHC, this channel is especially well suited to study jet scaling, including a proper experimental and theoretical error analysis. Combined with more channels where we expect staircase scaling, such a comprehensive study will not only provide crucial information on MC validation but will also help to eventually reveal the origin of staircase scaling.

A translation of these photon measurements into $W / Z+$ jets production is straightforward and will significantly impact new physics searches: typically, $\gamma+$ jets production is used to infer $(Z \rightarrow \nu \bar{\nu})+$ jets production in both control and signal regions [66-68]. Therefore, a better understanding of exclusive jet quantities and the translation of $\gamma+$ jets into $(Z \rightarrow \nu \bar{\nu})+$ jets production can help to systematically reduce the uncertainty of the background extrapolation in e.g. the jets+missing energy channel.

\section{Acknowledgments}

We are grateful to Alex Tapper for pointing us to this channel. The simulations underlying this study have been performed in parts on bwGRiD, member of the German D-Grid initiative, funded by the Bundesministerium für Bildung und Forschung and the Ministerium für Wissenschaft, Forschung und Kunst Baden-Württemberg.

Open Access. This article is distributed under the terms of the Creative Commons Attribution License which permits any use, distribution and reproduction in any medium, provided the original author(s) and source are credited.

\section{References}

[1] ATLAS collaboration, Search for the Higgs boson in the $H \rightarrow W W \rightarrow$ lnln decay mode with the ATLAS Detector, ATLAS-CONF-2011-111 (2011).

[2] CMS collaboration, Search for the Higgs Boson in the Fully Leptonic $W^{+} W^{-}$Final State, CMS-HIG-11-003.

[3] D.E. Morrissey, T. Plehn and T.M. Tait, Physics searches at the LHC, arXiv:0912.3259 [INSPIRE].

[4] D.L. Rainwater, D. Zeppenfeld and K. Hagiwara, Searching for $H \rightarrow \tau \tau$ in weak boson fusion at the CERN LHC, Phys. Rev. D 59 (1998) 014037 [hep-ph/9808468] [INSPIRE].

[5] T. Plehn, D.L. Rainwater and D. Zeppenfeld, A Method for identifying $H \rightarrow \tau \tau \rightarrow e^{ \pm} \mu^{\mp}$ missing $p_{T}$ at the CERN LHC, Phys. Rev. D 61 (2000) 093005 [hep-ph/9911385] [INSPIRE].

[6] S. Asai et al., Prospects for the search for a standard model Higgs boson in ATLAS using vector boson fusion, Eur. Phys. J. C 32S2 (2004) 19 [hep-ph/0402254] [INSPIRE]. 
[7] U. Baur and E. Glover, Tagging the Higgs boson in $p p \rightarrow W^{+} W^{-} j j$, Phys. Lett. B 252 (1990) 683 [INSPIRE].

[8] V.D. Barger, K.-m. Cheung, T. Han and D. Zeppenfeld, Single forward jet tagging and central jet vetoing to identify the leptonic $W W$ decay mode of a heavy Higgs boson, Phys. Rev. D 44 (1991) 2701 [Erratum ibid. D 48 (1993) 5444] [INSPIRE].

[9] V.D. Barger, R. Phillips and D. Zeppenfeld, Mini-jet veto: A Tool for the heavy Higgs search at the LHC, Phys. Lett. B 346 (1995) 106 [hep-ph/9412276] [INSPIRE].

[10] D.L. Rainwater, R. Szalapski and D. Zeppenfeld, Probing color singlet exchange in $Z+$ two jet events at the CERN LHC, Phys. Rev. D 54 (1996) 6680 [hep-ph/9605444] [INSPIRE].

[11] D.L. Rainwater, D. Summers and D. Zeppenfeld, Multi-jet structure of high E(T) hadronic collisions, Phys. Rev. D 55 (1997) 5681 [hep-ph/9612320] [InSPIRE].

[12] E. Gerwick, T. Plehn and S. Schumann, Understanding Jet Scaling and Jet Vetos in Higgs Searches, Phys. Rev. Lett. 108 (2012) 032003 [arXiv: 1108.3335] [INSPIRE].

[13] S. Ask, J. Collins, J. Forshaw, K. Joshi and A. Pilkington, Identifying the colour of TeV-scale resonances, JHEP 01 (2012) 018 [arXiv:1108.2396] [INSPIRE].

[14] C. Englert, T. Plehn, P. Schichtel and S. Schumann, Jets plus Missing Energy with an Autofocus, Phys. Rev. D 83 (2011) 095009 [arXiv:1102.4615] [INSPIRE].

[15] T. Plehn, D. Rainwater and P.Z. Skands, Squark and gluino production with jets, Phys. Lett. B 645 (2007) 217 [hep-ph/0510144] [INSPIRE].

[16] T. Plehn and T.M. Tait, Seeking Sgluons, J. Phys. G 36 (2009) 075001 [arXiv:0810.3919] [INSPIRE].

[17] J. Alwall, S. de Visscher and F. Maltoni, QCD radiation in the production of heavy colored particles at the LHC, JHEP 02 (2009) 017 [arXiv:0810.5350] [INSPIRE].

[18] ATLAS collaboration, G. Aad et al., Measurement of multi-jet cross sections in proton-proton collisions at a 7 TeV center-of-mass energy, Eur. Phys. J. C 71 (2011) 1763 [arXiv:1107.2092] [INSPIRE].

[19] M.E. Peskin and D.V. Schroeder, An Introduction to quantum field theory, Addison-Wesley, Reading U.S.A (1995).

[20] S. Ellis, R. Kleiss and W. Stirling, W's, Z's and Jets, Phys. Lett. B 154 (1985) 435 [INSPIRE].

[21] F.A. Berends, W. Giele, H. Kuijf, R. Kleiss and W. Stirling, Multi-jet production in W, Z events at $p \bar{p}$ colliders, Phys. Lett. B 224 (1989) 237 [InSPIRE].

[22] F.A. Berends, H. Kuijf, B. Tausk and W. Giele, On the production of a $W$ and jets at hadron colliders, Nucl. Phys. B 357 (1991) 32 [inSPIRE].

[23] CMS collaboration, S. Chatrchyan et al., Jet Production Rates in Association with $W$ and $Z$ Bosons in pp Collisions at $\sqrt{s}=7 \mathrm{TeV}$, arXiv:1110.3226 [INSPIRE].

[24] CMS collaboration, Study of $Z$ production in association with jets in proton-proton collisions at $\sqrt{s}=10$ TeV with the CMS detector at the CERN LHC, CMS-PAS-EWK-08-006.

[25] ATLAS collaboration, G. Aad et al., Search for new phenomena in final states with large jet multiplicities and missing transverse momentum using $\sqrt{s}=7$ TeV pp collisions with the ATLAS detector, JHEP 11 (2011) 099 [arXiv:1110.2299] [INSPIRE]. 
[26] V. Gribov and L. Lipatov, Deep inelastic ep scattering in perturbation theory, Sov. J. Nucl. Phys. 15 (1972) 438 [INSPIRE].

[27] G. Altarelli and G. Parisi, Asymptotic Freedom in Parton Language, Nucl. Phys. B 126 (1977) 298 [INSPIRE].

[28] Y.L. Dokshitzer, Calculation of the Structure Functions for Deep Inelastic Scattering and $e^{+} e^{-}$Annihilation by Perturbation Theory in Quantum Chromodynamics., Sov. Phys. JETP 46 (1977) 641 [INSPIRE].

[29] I.W. Stewart and F.J. Tackmann, Theory Uncertainties for Higgs and Other Searches Using Jet Bins, arXiv:1107.2117 [inSPIRE].

[30] M. Bengtsson and T. Sjöstrand, Coherent Parton Showers Versus Matrix Elements: Implications of PETRA - PEP Data, Phys. Lett. B 185 (1987) 435 [INSPIRE].

[31] G. Miu and T. Sjöstrand, W production in an improved parton shower approach, Phys. Lett. B 449 (1999) 313 [hep-ph/9812455] [INSPIRE].

[32] M.H. Seymour, A Simple prescription for first order corrections to quark scattering and annihilation processes, Nucl. Phys. B 436 (1995) 443 [hep-ph/9410244] [INSPIRE].

[33] G. Corcella and M.H. Seymour, Initial state radiation in simulations of vector boson production at hadron colliders, Nucl. Phys. B 565 (2000) 227 [hep-ph/9908388] [INSPIRE].

[34] S. Catani, F. Krauss, R. Kuhn and B. Webber, QCD matrix elements + parton showers, JHEP 11 (2001) 063 [hep-ph/0109231] [INSPIRE].

[35] S. Hoeche, F. Krauss, S. Schumann and F. Siegert, QCD matrix elements and truncated showers, JHEP 05 (2009) 053 [arXiv:0903.1219] [INSPIRE].

[36] M.L. Mangano, M. Moretti and R. Pittau, Multijet matrix elements and shower evolution in hadronic collisions: $W b \bar{b}+n$ jets as a case study, Nucl. Phys. B 632 (2002) 343 [hep-ph/0108069] [INSPIRE].

[37] T. Plehn, Lectures on LHC Physics, arXiv:0910.4182 [INSPIRE].

[38] J.R. Andersen, L. Lönnblad and J.M. Smillie, A Parton Shower for High Energy Jets, JHEP 07 (2011) 110 [arXiv: 1104.1316] [INSPIRE].

[39] J. Forshaw, J. Keates and S. Marzani, Jet vetoing at the LHC, JHEP 07 (2009) 023 [arXiv:0905.1350] [INSPIRE].

[40] R.M.D. Delgado, J.R. Forshaw, S. Marzani and M.H. Seymour, The dijet cross section with a jet veto, JHEP 08 (2011) 157 [arXiv:1107.2084] [INSPIRE].

[41] T. Gleisberg et al., Event generation with SHERPA 1.1, JHEP 02 (2009) 007 [arXiv:0811.4622] [INSPIRE].

[42] S. Schumann and F. Krauss, A Parton shower algorithm based on Catani-Seymour dipole factorisation, JHEP 03 (2008) 038 [arXiv:0709.1027] [INSPIRE].

[43] T. Gleisberg and S. Hoeche, Comix, a new matrix element generator, JHEP 12 (2008) 039 [arXiv:0808.3674] [INSPIRE].

[44] ATLAS collaboration, G. Aad et al., Measurement of the production cross section for $W$-bosons in association with jets in pp collisions at $\sqrt{s}=7 \mathrm{TeV}$ with the ATLAS detector, Phys. Lett. B 698 (2011) 325 [arXiv:1012.5382] [INSPIRE]. 
[45] THE ATLAS collaboration, S. Ask, Measurement of the production cross section for $W$ - and Z-bosons in association with jets in ATLAS, arXiv:1106.2061 [INSPIRE].

[46] ATLAS collaboration, G. Aad et al., Measurement of dijet production with a veto on additional central jet activity in pp collisions at $\sqrt{s}=7 \mathrm{TeV}$ using the ATLAS detector, JHEP 09 (2011) 053 [arXiv:1107.1641] [INSPIRE].

[47] ATLAS collaboration, G. Aad et al., Measurement of multi-jet cross sections in proton-proton collisions at a 7 TeV center-of-mass energy, Eur. Phys. J. C 71 (2011) 1763 [arXiv:1107.2092] [INSPIRE].

[48] CMS collaboration, S. Chatrchyan et al., Measurement of the Ratio of the 3-jet to 2-jet Cross sections in pp Collisions at $\sqrt{s}=7$ TeV, Phys. Lett. B 702 (2011) 336 [arXiv: 1106.0647] [INSPIRE].

[49] CMS collaboration, S. Chatrchyan et al., Jet Production Rates in Association with $W$ and $Z$ Bosons in pp Collisions at $\sqrt{s}=7 \mathrm{TeV}$, arXiv:1110.3226 [INSPIRE].

[50] D0 collaboration, V. Abazov et al., Measurement of the ratios of the $Z / \gamma^{*}+\geq n$ jet production cross sections to the total inclusive $Z /$ gamma $^{*}$ cross section in p $\bar{p}$ collisions at $\sqrt{s}=1.96 \mathrm{TeV}$, Phys. Lett. B 658 (2008) 112 [hep-ex/0608052] [INSPIRE].

[51] CDF - Run II collaboration, T. Aaltonen et al., Measurement of inclusive jet cross-sections in $Z / \gamma^{*} \rightarrow e^{+} e^{-}+$jets production in $p \bar{p}$ collisions at $\sqrt{s}=1.96$ TeV, Phys. Rev. Lett. 100 (2008) 102001 [arXiv:0711.3717] [INSPIRE].

[52] C. Berger et al., Precise Predictions for $W+4$ Jet Production at the Large Hadron Collider, Phys. Rev. Lett. 106 (2011) 092001 [arXiv: 1009.2338] [INSPIRE].

[53] M. Cacciari and G.P. Salam, Dispelling the $N^{3}$ myth for the $k_{t}$ jet-finder, Phys. Lett. B 641 (2006) 57 [hep-ph/0512210] [INSPIRE].

[54] M. Cacciari, G. P. Salam and G. Soyez, http://fastjet.fr.

[55] M. Cacciari, G.P. Salam and G. Soyez, The Anti-kt jet clustering algorithm, JHEP 04 (2008) 063 [arXiv: 0802.1189] [INSPIRE].

[56] M. Escalier et al., Photon/jet separation with DC1 data, PHYS-PUB-2005-018.

[57] U. Baur, T. Han and J. Ohnemus, QCD corrections to hadronic $W \gamma$ production with nonstandard $W W \gamma$ couplings, Phys. Rev. D 48 (1993) 5140 [hep-ph/9305314] [INSPIRE].

[58] S. Frixione, Isolated photons in perturbative QCD, Phys. Lett. B 429 (1998) 369 [hep-ph/9801442] [INSPIRE].

[59] S. Hoeche, S. Schumann and F. Siegert, Hard photon production and matrix-element parton-shower merging, Phys. Rev. D 81 (2010) 034026 [arXiv:0912.3501] [INSPIRE].

[60] F. Campanario, C. Englert and M. Spannowsky, Precise predictions for (non-standard) $W \gamma$ + jet production, Phys. Rev. D 83 (2011) 074009 [arXiv:1010.1291] [INSPIRE].

[61] J.M. Campbell, R. Ellis and C. Williams, Vector boson pair production at the LHC, JHEP 07 (2011) 018 [arXiv: 1105.0020] [INSPIRE].

[62] H.-L. Lai et al., New parton distributions for collider physics, Phys. Rev. D 82 (2010) 074024 [arXiv: 1007.2241] [INSPIRE].

[63] S. Catani, Y.L. Dokshitzer, M. Olsson, G. Turnock and B. Webber, New clustering algorithm for multi-jet cross-sections in $e^{+} e^{-}$annihilation, Phys. Lett. B 269 (1991) 432 [INSPIRE]. 
[64] B.R. Webber, QCD Jets and Parton Showers, arXiv:1009.5871 [InSPIRE].

[65] CMS collaboration, V. Khachatryan et al., Search for Supersymmetry in pp Collisions at $7 \mathrm{TeV}$ in Events with Jets and Missing Transverse Energy, Phys. Lett. B 698 (2011) 196 [arXiv:1101.1628] [INSPIRE].

[66] CMS collaboration, Data-Driven Estimation of the Invisible Z Background to the SUSY MET Plus Jets Search, CMS PAS SUS-08-002.

[67] Z. Bern et al., Driving Missing Data at Next-to-Leading Order, Phys. Rev. D 84 (2011) 114002 [arXiv: 1106.1423] [INSPIRE].

[68] S. Ask, M. Parker, T. Sandoval, M. Shea and W. Stirling, Using gamma+jets Production to Calibrate the Standard Model Z(nunu)+jets Background to New Physics Processes at the LHC, JHEP 10 (2011) 058 [arXiv:1107.2803] [INSPIRE]. 